\title{
BOUNDARY INTERPOLATION AND APPROXIMATION BY INFINITE BLASCHKE PRODUCTS
}

\author{
ISABELLE CHALENDAR, PAMELA GORKIN and JONATHAN R. PARTINGTON
}

\begin{abstract}
This paper considers the problem of boundary interpolation (in the sense of non-tangential limits) by Blaschke products and interpolating Blaschke products. Simple and constructive proofs, which also work in the more general situation of $H^{\infty}(\Omega)$ where $\Omega$ is a more general domain, are given of a number of results showing the existence of Blaschke products solving certain interpolation problems at a countable set of points on the circle. A variant of Frostman's theorem is also presented.
\end{abstract}

\section{Introduction}

It is a well known theorem of Fatou that a bounded analytic function $f$ on the open unit disc $D$ has radial limits at almost all points of the unit circle. In addition, each bounded analytic function can be factored in a natural way into a product of an inner function and an outer function. Thus, the study of the space of bounded analytic functions, $H^{\infty}$, is greatly aided by studying each of these entities separately. Because of the importance of inner functions as fundamental building blocks for analytic functions, there has been an extensive amount of work on them. Each inner function is created using fundamental building blocks as well: every inner function is a product of a Blaschke product and a singular inner function. In this paper we focus on Blaschke products.

A Blaschke product is an inner function $B$ of the form

$$
B(z)=\lambda \prod_{j=1}^{\infty} \frac{\left|z_{j}\right|}{z_{j}} \frac{z_{j}-z}{1-\overline{z_{j}} z},
$$

where $z_{j} \in \mathrm{D}$ and $|\lambda|=1$. In interpolation theory, Blaschke products play a particularly important role. For example, the Nevanlinna-Pick theorem says that if we are given a finite set of points $z_{1}, \ldots, z_{n}$ in $\mathrm{D}$, then there exists a solution to the interpolation problem $f\left(z_{j}\right)=w_{j}$ for $j=1, \ldots, n$ with $f \in H^{\infty}$ and $\|f\|_{\infty} \leq 1$, if and only if a certain quadratic form is nonnegative.

Received 1 July 2009, in revised form 12 October 2009. 
When this happens, there is a (finite) Blaschke product of degree at most $n$ solving the interpolation problem. It is possible to generalize this work in many ways: the finite set of points $\left\{z_{1}, \ldots, z_{n}\right\}$ can be replaced by interpolating sequences $\left(z_{n}\right)$ inside the unit disc, one can ask when functions in a space other than $H^{\infty}$ do the interpolation, and in the case in which the function has radial limits it is possible to consider boundary interpolation. In this paper, we will focus on boundary interpolation by Blaschke products.

While it may not make sense to study the value of the Blaschke product on the unit circle, it certainly makes sense to study radial limits of Blaschke products wherever they exist. Finite Blaschke products, for example, are continuous everywhere on the unit circle. Thus, asking when points $z_{1}, \ldots, z_{n}$ on the unit circle can be mapped to a second set of points on the unit circle by a finite Blaschke product makes sense and many results in this direction exist. In fact, assuming the $z_{j}$ are distinct, it turns out that interpolation is always possible and the Blaschke product is never unique (see, for example, [5], [22], and [16]). For infinite sequences $\left(z_{j}\right)$ the situation is quite intriguing. Letting $B^{*}(z)$ denote the radial limit of the Blaschke product $B$ at a point $z$ on the unit circle, G. T. Cargo [6] showed that given $n$ distinct points $z_{1}, \ldots, z_{n}$ on the unit circle and $n$ points $w_{1}, \ldots, w_{n}$ inside the unit disc, there exists a Blaschke product $B$ such that $B^{*}\left(z_{j}\right)=w_{j}$ for each $j$. In [3], Belna, Cowell, and Piranian extended this result to (infinite) sequences of points. A Blaschke product is said to be an interpolating Blaschke product if its zero sequence $\left(z_{n}\right)$ has the property that there exists a positive constant $\delta$ such that

$$
\inf _{m \neq n} \prod\left|\frac{z_{m}-z_{n}}{1-\overline{z_{m}} z_{n}}\right| \geq \delta>0 .
$$

In [12], the second author and Mortini showed that, under appropriate conditions, interpolation can also be accomplished using an interpolating Blaschke product. Later, Hjelle [19] also gave a constructive proof of this result. In [14], the authors studied interpolation by functions of minimal norm and noted that the techniques in the aforementioned paper can be applied to more general domains. The same is true for some of the proofs in Section 3 of this paper.

We begin by considering relevant interpolation results. Given two finite sets of interlaced points on the unit circle (where the points are ordered in terms of increasing argument) the authors of [16] gave an algorithm that produced a Blaschke product of lowest degree that identifies each set of points. In Section 2, we show that under certain convergence assumptions, the same can be done for infinitely many points. In Section 3, we also look at interpolation on the unit circle. We show that if we are given a uniformly bounded sequence of analytic functions, $\left(p_{n}\right)$, then we can approximate these functions (along radii) 
with a function of norm $\sup _{n}\left\|\left(p_{n}\right)\right\|_{\infty}$. In case the $p_{n}$ are constants this result can, for example, be deduced from a theorem of Belna, Cowell, and Piranian (Theorem 3.1). However, our techniques are taken from the theory of uniform algebras and therefore are potentially more widely applicable. As a corollary, in the case sup $\left\|p_{n}\right\|=1$, we show how to obtain a short proof that there exist Blaschke products that do the interpolation, obtaining a very special case of a result of Nicolau [24].

Since the radius is a connected set, its closure in the maximal ideal space of $H^{\infty}$ is a closed and connected set. Thus, given any inner function $I$, its radial cluster set is a compact, connected set. Theorem 3.1 tells us that given a sequence of points $\left(z_{n}\right)$ on the unit circle, there exists a Blaschke product $B$ such that the radial cluster set of $B$ is the same as the radial cluster set of $I$ at each $z_{n}$. This result was extended by R. Berman [4] and A. Nicolau [24]. In particular, Nicolau considered radial limits of more general functions on sets of Lebesgue measure zero and of type $F_{\sigma}$ and $G_{\delta}$. In Section 4, we prove a generalized Frostman's theorem. We will show the following: Given an inner function $I$ and a Blaschke product $b$, for almost all $\alpha$ sufficiently small, the function $I_{\alpha}=(I+\alpha(1-b)) /(b+\bar{\alpha}(b-1) I)$ is a ratio of Blaschke products. This fraction retains properties of the Blaschke product $b$ : if $b$ is interpolating, we can choose $\alpha$ so that the denominator is interpolating; if $b$ is thin, we can choose $\alpha$ so that the denominator is thin; if $b=1$ on a set $E$, then the fraction $I_{\alpha}$ will provide a Blaschke product approximating $I$ on the set $E$ (see, for example, [11, Chapter X, section 5] and [29] for a discussion of results on approximation by ratios of interpolating Blaschke products). Since we may choose our interpolating Blaschke products tending to 1 along various subsets in $\mathrm{D}$, we recover information about the limiting behavior of $I$ along those subsets.

\section{Constructive interpolation by Blaschke products}

We begin with an infinite version of Theorem 8 from [8]. With additional assumptions on convergence it is possible to write down a simple and explicit expression for a Blaschke product that interpolates (certain sets of) infinitely many points on the unit circle. This is the first of our set of interpolation results. Two sets of real numbers $\left\{a_{1}, \ldots, a_{n}\right\}$ and $\left\{b_{1}, \ldots, b_{n}\right\}$ are said to be interlaced, if $a_{1}<b_{1}<\cdots<a_{n}<b_{n}$.

We begin with an easily-proved result that achieves interpolation in the simplest case.

THEOREM 2.1. Let $a_{1}<b_{1}<a_{2}<b_{2}<\cdots$ be a sequence of real numbers 
with limit 0 and satisfying the condition

$$
\sum \frac{\left|a_{k}-b_{k}\right|}{\left|a_{k}\right|}<\infty
$$

Then the infinite product $F(z)=\prod_{k=1}^{\infty} \frac{z-b_{k}}{z-a_{k}}$ converges locally uniformly to an analytic function on $\mathrm{C} \backslash\left\{a_{k}: k \geq 1\right\}$ such that $F$ maps the upper half-plane $\mathrm{H}^{+}$ into the upper half-plane, the lower half-plane $H^{-}$into the lower half-plane, and the extended real line into the extended real line (where the value at 0 is interpreted as a non-tangential limit).

Proof. Writing $F_{n}(z)=\prod_{k=1}^{n} \frac{z-b_{k}}{z-a_{k}}$, we have that $F_{n}$ maps $H^{ \pm}$to $H^{ \pm}$by [8, Thm. 8], and $F_{n}$ tends locally uniformly to $F$ because

$$
F_{n}(z)=\prod_{k=1}^{n}\left(1+\left(a_{k}-b_{k}\right) /\left(z-a_{k}\right)\right)
$$

and $\sum\left|a_{k}-b_{k}\right| /\left|z-a_{k}\right|$ converges locally uniformly. Also note that by equation (1), the sequence $\left(F_{n}(0)\right)$ converges. Now given $0<\epsilon<1$ choose $N$ such that

$$
\sum_{k=N+1}^{\infty} \frac{\left|a_{k}-b_{k}\right|}{\left|a_{k}\right|}<\epsilon .
$$

For every $\zeta$ purely imaginary and sufficiently close to 0 we have

$$
\left|\prod_{k=1}^{N} \frac{\zeta-b_{k}}{\zeta-a_{k}}-F_{N}(0)\right|<\epsilon
$$

To estimate the value of $F(\zeta)$ write $w_{k}=\left(a_{k}-b_{k}\right) /\left(\zeta-a_{k}\right)$. Note that since $\zeta$ is purely imaginary and $a_{k}$ is real we have

$$
\sum_{k=N+1}^{\infty}\left|w_{k}\right|=\sum_{k=N+1}^{\infty} \frac{\left|a_{k}-b_{k}\right|}{\left|\zeta-a_{k}\right|} \leq \sum_{k=N+1}^{\infty} \frac{\left|a_{k}-b_{k}\right|}{\left|a_{k}\right|}<\epsilon
$$

Therefore,

$$
\begin{aligned}
\left|\prod_{k=N+1}^{\infty}\left(1+w_{k}\right)-1\right| & \leq \prod_{k=N+1}^{\infty}\left(1+\left|w_{k}\right|\right)-1 \leq \exp \left(\sum_{k=N+1}^{\infty}\left|w_{k}\right|\right)-1 \\
& <e^{\epsilon}-1 \leq(e-1) \epsilon
\end{aligned}
$$

This implies that the non-tangential limit of $F(\zeta)$ has imaginary part of at most order $\epsilon$. Combining this fact with equation (2) and the convergence of $\left(F_{n}(0)\right)$, we see that the nontangential limit exists at 0 . 
The next result is a stronger form of Theorem 2.1.

THeORem 2.2. Let $\left(b_{k}\right)_{k \geq 1}$ be a sequence of distinct real numbers. Then there exists a sequence $c_{1}, a_{2}, a_{3}, \ldots$ of real numbers such that the product

$$
F(z)=\frac{z-c_{1}}{z-b_{1}} \prod_{k=2}^{\infty} \frac{z-b_{k}}{z-a_{k}}
$$

converges locally uniformly to a holomorphic function on $\mathrm{H}^{+} \cup \mathrm{H}^{-}$such that $F$ maps $H^{+}$into $H^{+}$and $H^{-}$into $H^{-}$. Further $F\left(b_{1}\right)=\infty$ and $F\left(b_{k}\right)=0$ for $k>1$, in the sense of non-tangential limits. Moreover, if the closure of $\left\{b_{k}\right\}_{k \geq 1}$ is countable, then $F$ maps the extended real line into the extended real line, in the sense of non-tangential limits.

Proof. Inductively we form a product

$$
F_{N}(z)=\frac{z-c_{1}}{z-b_{1}} \prod_{k=2}^{N} \frac{z-b_{k}}{z-a_{k}},
$$

for $N=2,3, \ldots$, such that at each stage the ordered zeroes $z_{1}, \ldots, z_{N}$, say, and poles $p_{1}, \ldots, p_{N}$, say, are interlaced, that is, $p_{1}<z_{1}<p_{2}<z_{2}<\ldots<$ $p_{N}<z_{N}$. It is clear that to do this it is enough at the $N$ th stage to choose $a_{N}$ sufficiently close to $b_{N}$ but distinct from all previously chosen points and not equal to any of the $\left(b_{k}\right)$, with an appropriate choice of sign of $a_{N}-b_{N}$. This guarantees that $F_{N}$ maps each of $H^{+}$and $H^{-}$to itself, by [8, Thm. 8], and preserves the extended real line.

In order that the limit function may exist and have the required properties, we choose our $N$-th point carefully: we retain the first $N-1$ points and we choose $a_{N}$ to satisfy the additional conditions $\left|b_{N}-a_{N}\right|<2^{-N},\left|a_{N}-b_{k}\right|>$ $N^{2}\left|b_{N}-a_{N}\right|$ and $\left|a_{N}-a_{k}\right|>N^{2}\left|b_{N}-a_{N}\right|$ for all $k<N$, and (in the case when the set of accumulation points of the set of $\left(b_{k}\right)$ is countable, including additional points $\ell_{1}, \ell_{2}, \ldots$ not already in the set of $\left(b_{k}\right)$ or $\left.\left\{c_{1}, a_{2}, \ldots, a_{N}\right\}\right)$, $\left|a_{N}-\ell_{k}\right|>N^{2}\left|b_{N}-a_{N}\right|$ for all $k<N$. This is always possible at this stage, because we have only finitely many points.

As in the proof of Theorem 2.1, we have local uniform convergence of the infinite product and, since the choice of the $\left(a_{N}\right)$ gives us that

$$
\sum_{j=2}^{\infty} \frac{\left|a_{j}-b_{j}\right|}{\left|a_{j}-z\right|}<\infty
$$

for all $z$ that are either (i) not in the closure of the set of $\left(b_{k}\right)$, (ii) one of the $a_{k}$ (where we exclude one term from the sum) or $b_{k}$, or (iii) in the closure of the 
set of $\left(b_{k}\right)$ if it is countable (i.e., including additional points $\left(\ell_{k}\right)$ as above), we deduce that $F$ is a holomorphic function mapping $\mathrm{H}^{+}$to $\mathrm{H}^{+}$and $\mathrm{H}^{-}$to $\mathrm{H}^{-}$, and the nontangential limit of $F$ at the points listed above is real (or infinite at the points $a_{k}$ and $b_{1}$ ).

In what follows, we let $\delta_{m n}=1$ if $m=n$ and 0 if $m \neq n$. For $z \in \partial \mathrm{D}$, we let $G^{*}(z)$ denote the radial limit of the function $G$ at the point $z$.

COROLlaRY 2.3. Let $\left(z_{n}\right)$ be a sequence of distinct points on the unit circle. Then for each $n$ there exists a function $G_{n} \in H^{\infty}$ with $\left\|G_{n}\right\|_{\infty} \leq 1$, such that $G_{n}^{*}\left(z_{m}\right)=(-1)^{\delta_{m n}+1}$. If the sequence has countable closure, then we may take $G_{n}$ to be a Blaschke product.

Proof. We transform to the half-plane and use Theorem 2.2. Note that a real or infinite nontangential limit at a real point now corresponds to a nontangential limit of modulus one at a point on the circle. Moreover, an inner function whose nontangential limits all have modulus one is necessarily a Blaschke product.

\section{Interpolation by inner functions}

Letting $C_{\rho}(B, z)$ denote the radial cluster set of a Blaschke product at the point $z$, we have the following result due to Belna, Cowell and Piranian.

THEOREM 3.1 ([3]). Let $\left(z_{n}\right)$ be a sequence of distinct points on the unit circle and let $\left(\Omega_{n}\right)$ denote a countable set of nonempty, closed, connected sets in $\overline{\mathrm{D}}$. Then there is a Blaschke product $B$ such that

$$
C_{\rho}\left(B, z_{n}\right)=\Omega_{n}
$$

for $n=1,2,3, \ldots$

Related results appear in [24] and [4]. In [24], Nicolau showed that given a set $E \subset \partial \mathrm{D}$ of measure zero and of type $F_{\sigma}$ and $G_{\delta}$, and a function $\phi$ defined on $E$ with $\sup \left\{\left|\phi\left(e^{i t}\right)\right|: e^{i t} \in E\right\} \leq 1$ such that for each open set $O$ the set $\phi^{-1}(O)$ is an $F_{\sigma}$ and $G_{\delta}$ set, then there is a Blaschke product $B$ that extends analytically to $\partial \mathrm{D} \backslash \bar{E}$ such that $\lim _{r \rightarrow 1^{-}} B\left(r e^{i t}\right)=\phi\left(e^{i t}\right)$ for $e^{i t} \in E$, and Nicolau shows that the result of Belna, Colwell and Piranian can be obtained as well. In [4], Berman considers the special case in which $\phi=0$ and $\phi=1$.

The papers [12], [13], and [19] study similar situations, but consider the existence of interpolating Blaschke products that do the interpolation. These proofs are primarily constructive and (necessarily) somewhat complicated. However, as we show below, our results from the previous section can be used to obtain a very simple proof of the existence of a Blaschke product that does the interpolation (see Corollary 3.6). Our proofs can be adapted to work in 
more general situations than previous proofs, because they rely on properties that many uniform algebras have. The next theorem is an auxiliary one, that will assist us in our simplified proof. While it follows from Cargo's or Belna, Cowell, and Piranian's result the following proof requires little machinery and obtains information about the subproducts. Since we wish to make our construction as general as possible, we include this proof below.

THEOREM 3.2. Let $\left(z_{n}\right)$ be a sequence of points on the unit circle. Then for each $n$, there exists a Blaschke product $B_{n}$ such that $B_{n}^{*}\left(z_{m}\right)=0$ if $m \neq n$ and $B_{n}^{*}\left(z_{n}\right)=1$. Furthermore, the Blaschke product can be chosen so that every subproduct of $B_{n}$ will have radial limit of modulus 1 at $z_{n}$.

Proof. Note that for each point $\lambda$, it is relatively easy to construct a Blaschke product $B_{\lambda}$ with radial limit 0 at $\lambda$ and radial limit of modulus 1 at every other point: To see this, look at the radius ending at $\lambda=e^{i \theta}$. Choose a sequence of points $\left(a_{j}\right)$ on the radius that are (equally) $\rho$-separated from each other, say $\rho\left(a_{j}, a_{j+1}\right)=1 / 2$. Now, these will form an interpolating Blaschke sequence [21, p. 204]. If we denote this set of points by $\left(a_{n}\right)$, then $\sum_{n}\left(1-\left|a_{n}\right|\right)<\infty$. Thus, we may choose a sequence $N_{n} \rightarrow \infty$ so that $\sum_{n} N_{n}\left(1-\left|a_{n}\right|\right)<\infty$. Note that for each $k$, we may drop off finitely many terms to assume that $\sum_{n} N_{n}\left(1-\left|a_{n}\right|\right)<1 / 2^{k}$. Now consider the Blaschke product $B_{\lambda}$ defined as follows:

$$
B_{\lambda}(z)=\prod_{j=1}^{\infty}\left(\frac{\left|a_{j}\right|}{a_{j}} \frac{a_{j}-z}{1-\overline{a_{j}} z}\right)^{N_{j}} .
$$

We claim that $B_{\lambda}$ has the property that $B_{\lambda}\left(r e^{i \theta}\right) \rightarrow 0$ as $r \rightarrow 1$. So let $r e^{i \theta}$ be an arbitrary point on the radius and note that by our choice of $a_{j}$, there exists $a_{j_{0}}$ such that $\rho\left(r e^{i \theta}, a_{j_{0}}\right) \leq 1 / 2$. Therefore

$$
\left|B_{\lambda}\left(r e^{i \theta}\right)\right| \leq\left|\frac{a_{j_{0}}-r e^{i \theta}}{1-\overline{a_{j_{0}}} e^{i \theta}}\right|^{N_{j_{0}}} \leq 1 / 2^{N_{j_{0}}} \rightarrow 0
$$

as $r \rightarrow 1$, because $r \rightarrow 1$ implies $N_{j_{0}} \rightarrow \infty$. Since $B_{\lambda}$ is continuous at $e^{i \gamma}$ for $\gamma \neq \theta$, the assertion that $B_{\lambda}$ has radial limits of modulus 1 at every other point follows.

We suppose now that our sequence is $\left(z_{n}\right)$ with $z_{1}=1$ and we construct $B_{j}$ so that Frostman's condition [7, p. 14] is satisfied at $z=1$; that is, if $\left(a_{m j}\right)$ denotes the zeroes of $B_{j}$, then

$$
\sum \frac{1-\left|a_{m j}\right|^{2}}{\left|1-a_{m j}\right|}<1 / 2^{j}
$$


It is known that if $B_{j}$ satisfies this condition, then $B_{j}$ and all of its subproducts have radial limit at the point 1 . To create $B_{j}$, choose zeroes $\left(a_{m j}\right)$ along the radius tending to $z_{j}$ (for $j>1$ ) as in the first paragraph so that $\sum(1-$ $\left.\left|a_{m j}\right|\right)<1 / 2^{j}$ and the Frostman condition $\sum \frac{1-\left|a_{m j}\right|^{2}}{\left|1-a_{m j}\right|}<1 / 2^{j}$ is satisfied, with multiplicity included. Thus $B_{j}$ will have radial limit zero at each $z_{j}$ and it (and all of its subproducts) will have radial limit of modulus 1 at the point 1.

Now if we form the Blaschke product whose zero sequence is the union of the zero sequences of the $B_{j}$, it is clear that the radial limit at each $z_{j}$ is zero. Since this new Blaschke product satisfies the Frostman condition at $z=1$, it has radial limit of modulus 1 at $z=1$. Multiplying by a constant of modulus 1, we obtain the desired Blaschke product.

To obtain our Blaschke product, we chose the zeros equally spaced. However, it is clear that if we move the zeros along the radius very slightly with respect to the pseudohyperbolic metric, the radial limit will remain zero. Thus, if we multiply functions of the type obtained in the previous theorem, we obtain the following corollary.

COROllary 3.3. Given a sequence $\left(z_{n}\right)$ of distinct points on the unit circle, there exists a Blaschke product $B$ with simple zeros such that $B^{\star}\left(z_{n}\right)=0$ for all $n$.

It was pointed out to us by the referee that an explicit example can also be found in [27, p. 317, Problem 13].

To complete our proof of Theorem 3.5 we use the following lemmas. The first of these is from [2].

Lemma 3.4. Let $j$ be an integer, $j>1$. Let $a_{1}, \ldots, a_{j}, b_{1}, \ldots, b_{j}$ be nonnegative numbers. Suppose that $a_{k}+b_{k} \leq 1$ for $k=1, \ldots, j$. Then

$$
a_{1}+a_{2} b_{1}+a_{3} b_{1} b_{2}+\cdots+a_{j} b_{1} \cdots b_{j-1} \leq 1 .
$$

We have now completed the preliminary work for the proof of Theorem 3.5 that relies, primarily, on the existence of the $B_{j}$ below, uniform algebra properties, and a normal families argument. Thus, they can be applied to $H^{\infty}$ on more general domains in the disc. In addition, we can interpolate functions rather than scalars, if our sequence of functions is uniformly bounded.

THEOREM 3.5. Let $\left(e^{i \theta_{n}}\right)$ denote a sequence of points on the unit circle. Let $\left(k_{n}\right)$ be a sequence of analytic functions for which there exists a constant $M$ with sup $\left\|k_{n}\right\|_{\infty}=M$. Then there exists a function $F \in H^{\infty}$ for which $\lim _{r \rightarrow 1}\left|F\left(r e^{i \theta_{n}}\right)-k_{n}\left(r e^{i \theta_{n}}\right)\right|=0$ for each $n$. Further, we may choose the interpolating function $F$ so that $\|F\|_{\infty}=M$. 
Proof. Use Corollary 2.3 to obtain $H^{\infty}$ functions $G_{n}$ with $\left\|G_{n}\right\| \leq 1$ and $G_{n}^{*}\left(z_{m}\right)=(-1)^{\delta_{m n}+1}$. Let $g_{n}=\left(G_{n}+1\right)^{2} / 4$ and $h_{n}=\left(G_{n}-1\right)^{2} / 4$. Then

(1) $\left|g_{n}\right|+\left|h_{n}\right| \leq 1$ on $\mathrm{D}$;

(2) $g_{n}^{*}\left(z_{n}\right)=1$ and $g_{n}^{*}\left(z_{m}\right)=0$ for $m \neq n$;

(3) $h_{n}^{*}\left(z_{n}\right)=0$ and $h_{n}^{*}\left(z_{m}\right)=1$ for $m \neq n$.

Let

$$
F_{n}=k_{1} g_{1}+k_{2} g_{2} h_{1}+k_{3} g_{3} h_{1} h_{2}+\cdots+k_{n} g_{n} h_{1} \ldots h_{n-1} .
$$

Then, by Lemma 3.4, $\left\|F_{n}\right\|_{\infty} \leq \max \left\{\left\|k_{j}\right\|: j=1, \ldots, n\right\}$. Now fix $j$.

Note that $\left|F_{m}\left(r e^{i \theta_{j}}\right)-k_{j}\left(r e^{i \theta_{j}}\right)\right| \rightarrow 0$ for each $m$ and $j=1, \ldots, m$. If $n, m \geq N>j$ we use Lemma 3.4 to see that

$$
\begin{aligned}
\left|F_{n}\left(r e^{i \theta_{j}}\right)-F_{m}\left(r e^{i \theta_{j}}\right)\right| & \leq\left|\left(h_{1} \cdots h_{N-1}\right)\left(r e^{i \theta_{j}}\right)\right| \sup \left\{\left\|k_{j}\right\|: j \geq N\right\} \\
& \leq M\left|h_{j}\left(r e^{i \theta_{j}}\right)\right| .
\end{aligned}
$$

Recalling that $\lim _{r \rightarrow 1}\left|k_{j}\left(r e^{i \theta_{j}}\right)-F_{n}\left(r e^{i \theta_{j}}\right)\right|$ for $n>j$, and choosing $r \in \mathrm{R}$ with $0<r<1$, we use Lemma 3.4 once more to obtain

$$
\begin{aligned}
\left|F\left(r e^{i \theta_{j}}\right)-k_{j}\left(r e^{i \theta_{j}}\right)\right| \leq & \left|F\left(r e^{i \theta_{j}}\right)-F_{n}\left(r e^{i \theta_{j}}\right)\right|+\left|F_{n}\left(r e^{i \theta_{j}}\right)-k_{j}\left(r e^{i \theta_{j}}\right)\right| \\
\leq & M\left|h_{j}\left(r e^{i \theta_{j}}\right)\right|\left[\left|h_{1} h_{2} \cdots h_{j-1} h_{j+1} \cdots h_{n-1}\left(r e^{i \theta_{j}}\right)\right|\right] \\
& \quad+\left|F_{n}\left(r e^{i \theta_{j}}\right)-k_{j}\left(r e^{i \theta_{j}}\right)\right| \\
\leq & M\left|h_{j}\left(r e^{i \theta_{j}}\right)\right|+\left|F_{n}\left(r e^{i \theta_{j}}\right)-k_{j}\left(r e^{i \theta_{j}}\right)\right| .
\end{aligned}
$$

Our assumptions imply that $h_{j}\left(r e^{i \theta_{j}}\right) \rightarrow 0$ as $r \rightarrow 1$ and the second summand also tends to zero as $r \rightarrow 1$, completing the proof.

As a corollary, we obtain a very special case of Nicolau's result on radial limits of Blaschke products [24]. The proof is similar to the one in [14, p. 496] that was used to obtain functions that interpolate sequences in the maximal ideal space of $H^{\infty}$, as opposed to sequence of points on the boundary of the disc. (See also [28].)

Corollary 3.6. Given a sequence of distinct points on the unit circle, $\left(z_{j}\right)_{j}$, and a sequence of analytic functions $\left(k_{j}\right)$ defined on the unit disc such that sup $\left\|k_{j}\right\|=1$, there is a Blaschke product b such that $\left|b\left(r e^{i \theta_{j}}\right)-k_{j}\left(r e^{i \theta_{j}}\right)\right| \rightarrow 0$ for all $j$.

Proof. Choose a function $F$, using Theorem 3.5, that does the interpolation. Choose a Blaschke product $B$, according to Corollary 3.3 with radial limit 
0 at each $z_{j}$ and use Corollary 2.3 to obtain a second (nonconstant) function $G$ of norm 1 with radial limit 1 at each $z_{j}$. Consider the set

$$
\mathscr{S}=\left\{\left(\frac{1-G}{2}\right)^{2} B h+\left(\frac{1+G}{2}\right)^{2} F: h \in H^{\infty},\|h\| \leq 1\right\} .
$$

Let $I=F(1+G)^{2} / 4$. Letting $\xi_{n}$ denote the zeros of $B$, consider the interpolation problem $f\left(\xi_{n}\right)=I\left(\xi_{n}\right)$ where $f$ is in the (closed) unit ball of $H^{\infty}$. Then every function in $\mathscr{S}$ is a solution of this problem and therefore there are (at least) two distinct solutions to this interpolation problem. By Stray's theorem [28], there is a Blaschke solution $b$. Now $b-I=B k$ for some $k \in H^{\infty}$ and $B\left(r z_{j}\right) \rightarrow 0$ as $r \rightarrow 1$. Since

$$
b=I+B k=F(1+G)^{2} / 4+B k,
$$

$G\left(r z_{j}\right) \rightarrow 1$ and $B\left(r z_{j}\right) \rightarrow 0$ for each $j$, it follows that $b$ is the solution we seek.

ReMARK 3.7. Theorem 3.5 above is valuable because of its constructive nature. As Nicolau shows [24, p. 251] it follows easily from Berman's result [4] and Stray's theorem [28] that for each analytic function $g$ in the unit ball of $H^{\infty}$ there exists a Blaschke product $B$ that extends analytically to $\backslash \backslash \bar{E}$ such that

$$
\lim _{r \rightarrow 1}\left(I\left(r e^{i t}\right)-g\left(r e^{i t}\right)\right)=0
$$

for $e^{i t} \in E$, where $E$ is a set of measure zero that is of type $F_{\sigma}$ and $G_{\delta}$.

\section{A variation of Frostman's theorem}

In this section, we present a variant of Frostman's theorem. For a single point on the unit circle, S. Axler noted this in his paper [1].

To obtain our results, we will need one technical lemma later that is dependent upon the special nature of the so-called thin Blaschke products. Recall that a Blaschke product is said to be an interpolating Blaschke product if its zero sequence $\left(z_{n}\right)$ has the property that there exists a positive constant $\delta$ such that

$$
\inf _{m \neq n} \prod\left|\frac{z_{m}-z_{n}}{1-\overline{z_{m}} z_{n}}\right| \geq \delta>0 .
$$

This is a condition on the separation of the zeroes of the Blaschke product; that is, if we denote the pseudohyperbolic distance between two points $z$ and $w$ in the unit disc by $\rho$ (so that $\rho(z, w)=\left|\frac{z-w}{1-\bar{z} w}\right|$ ). The pseudohyperbolic disc of radius $\delta$ about a point $z$ will be denoted by $D_{\rho}(z, \delta)$. 
We will use the notion of the so-called thin Blaschke products: A Blaschke product is thin if

$$
\lim _{n \rightarrow \infty}\left(1-\left|z_{n}\right|^{2}\right)\left|B^{\prime}\left(z_{n}\right)\right|=1 .
$$

Note that a thin Blaschke product may have finitely many repeated zeroes. For interpolating Blaschke products we have the following well-known lemma of Hoffman ([20]). (The version we cite below can be found on [11, p. 404].)

Lemma 4.1 (Hoffman's Lemma). Let $B$ be an interpolating Blaschke product with zeroes $\left(z_{n}\right)$ satisfying

$$
\inf _{n}\left(1-\left|z_{n}\right|^{2}\right)\left|B^{\prime}\left(z_{n}\right)\right| \geq \delta>0 .
$$

Then there exist positive constants, $\lambda=\lambda(\delta)$ and $r=r(\delta)$, with $\lambda<1$, $\lambda<2 \lambda /\left(1+\lambda^{2}\right)<\delta$ and $r<1$, satisfying

$$
\begin{aligned}
& \lim _{\delta \rightarrow 1} \lambda(\delta)=1 \\
& \lim _{\delta \rightarrow 1} r(\delta)=1
\end{aligned}
$$

and the set $B^{-1}(\Delta(0, r))=\{z:|B(z)|<r\}$ is the union of pairwise disjoint domains $V_{n}, z_{n} \in V_{n}$, and

$$
V_{n} \subset\left\{z: \rho\left(z, z_{n}\right)<\lambda\right\} .
$$

Also, B maps each domain $V_{n}$ univalently onto $\Delta(0, r)=\{w:|w|<r\}$.

We now turn to the extension of Frostman's theorem. There are many theorems about subalgebras of $L^{\infty}$ that are related to Theorem 4.4 below; our result seems to combine several of them. Recall that $H^{\infty}+C$ is the closed subalgebra of $L^{\infty}$ containing functions that are sums of functions in $H^{\infty}$ and continuous functions. It is known [17] that for every inner function $I$ there is a Blaschke product $B$ such that $I / B=I \bar{B}$ and $B / I=B \bar{I}$ are both in $H^{\infty}+C$. Thus, there is a unimodular function $u$ for which $u$ and $\bar{u}$ are in $H^{\infty}+C$, and such that $B \bar{I}=u$. Therefore $B / u=I$ almost everywhere on $\partial \mathrm{D}$.

We will use the following result that is useful in many contexts (see, for example, [30, Lemma 5.4]).

Lemma 4.2. Let $\left(z_{n}\right)$ and $\left(z_{n}^{\prime}\right)$ be sequences satisfying $\sup \rho\left(z_{n}, z_{n}^{\prime}\right)<1$. Then $\left(z_{n}\right)$ is thin if and only if $\left(z_{n}^{\prime}\right)$ is thin.

Finally, we recall a result of S. D. Fisher and J. E. Shapiro:

THEOREM 4.3. [9] Let $f, g \in H^{\infty}$ (D) have no common singular inner factor. Then the set of $\alpha$ for which $f+\alpha g$ has a nontrivial singular inner factor has logarithmic capacity zero. 
Having completed the preliminaries, we turn to the theorem.

THEOREM 4.4. Let I be an inner function and let $b$ be a Blaschke product. Then there exists $\epsilon>0$ such that for all $\alpha$ with $|\alpha|<\epsilon$, except possibly a set of capacity zero,

$$
\frac{I+\alpha(1-b)}{b+\bar{\alpha}(b-1) I}=\frac{\tilde{b}}{b_{\alpha}}
$$

where $\tilde{b}$ and $b_{\alpha}$ are Blaschke products depending on $\alpha$. If the Blaschke product $b$ is interpolating, then $b_{\alpha}$ will be as well and if the Blaschke product $b$ is thin, then $b_{\alpha}$ will be too.

Proof. Let $I_{\alpha}=\frac{I+\alpha(1-b)}{b+\bar{\alpha}(b-1) I}$. Note that by Theorem 4.3, for all $\alpha$ except a set of capacity zero,

$$
b+\bar{\alpha}(b-1) I=b_{\alpha} g_{\alpha},
$$

where $b_{\alpha}$ is a Blaschke product and $g_{\alpha}$ is an outer function. Since $b$ is inner and $\alpha$ can be chosen to be small, we may further assume that $g_{\alpha}$ is invertible in $L^{\infty}$ and therefore in $H^{\infty}$. Similarly, for an appropriate choice of $\alpha$, we note that $1-b$ is outer and we again apply Theorem 4.3 to conclude that $I+\alpha(1-b)=\tilde{b} F_{\alpha}$, where $F_{\alpha}$ is an invertible outer function. Now $b_{\alpha} I_{\alpha} \in$ $H^{\infty}$ (D) and $\left|b_{\alpha} I_{\alpha}\right|=1$ almost everywhere on the unit circle, so it is inner. Thus, $b_{\alpha} I_{\alpha}=\tilde{b} F_{\alpha} / g_{\alpha}$ so $F_{\alpha} / g_{\alpha}$ is an outer function with modulus one almost everywhere on the unit circle and therefore it is constant.

Now suppose that the initial Blaschke product $b$ is interpolating. Let $\epsilon>0$ and consider $b+\epsilon(b-1) I$. Then for $\epsilon$ sufficiently small, there exists a Blaschke product $b_{\epsilon}$ that is a finite product of interpolating Blaschke products and an invertible outer function $F_{\epsilon}$ such that $b+\epsilon(b-1) I=b_{\epsilon} F_{\epsilon}$ (see, for example, [18]). Using Hoffman's lemma (Lemma 4.1) and the fact that we now assume that $b$ is interpolating, the zeros of $b_{\epsilon}$ for $\epsilon$ sufficiently small, will be $\rho$ separated. If we write $b_{\epsilon}=\prod_{j=1}^{n} b_{j}$ with $b_{j}$ interpolating, this means that there exists $\delta>0$ such that the pseudohyperbolic distance $\rho\left(Z_{\mathrm{D}}\left(b_{j}\right), Z_{\mathrm{D}}\left(b_{k}\right)\right) \geq \delta$ for $j \neq k$ and therefore the union is an interpolating sequence (see [20], [25, p. 217] or [11, p. 287]). Thus, $b_{\epsilon}$ will actually be interpolating.

To complete the proof, we now consider the case in which $b$ is thin. Let $\left(z_{n}\right)$ denote the zero sequence of the thin Blaschke product $b$. Using Lemma 4.1, we know that there exist $\delta$ and $\lambda$ such that $\{z:|b(z)|<\delta\} \subseteq \bigcup_{n} D_{\rho}\left(z_{n}, \lambda\right)$. Thus, if $w \notin D_{\rho}\left(z_{n}, \lambda\right)$ for any $n$, then $|b(z)| \geq \delta$. Now let $\alpha$ be chosen with $2|\alpha|<\delta$. Then

$$
\left|b_{\alpha}(z) g_{\alpha}(z)-b(z)\right|<2|\alpha| \text {. }
$$

In particular, $b_{\alpha}$ cannot vanish outside $\bigcup_{n} D_{\rho}\left(z_{n}, \lambda\right)$. Further, by Rouché's theorem, $b_{\alpha} g_{\alpha}$ has the same number of zeroes as $b$ in $D_{\rho}\left(z_{n}, \lambda\right)$. Because 
$b$ is thin, there exists $N$ such that $b$ has exactly one zero in $D_{\rho}\left(z_{n}, \lambda\right)$ for $n \geq N$. Thus, recalling that the zeroes of $b$ were denoted by $\left(z_{n}\right)$, and letting the zeroes of $b_{\alpha}$ be denoted by $\left(z_{n}^{\prime}\right)$, we see that these two sets of points satisfy the conditions of Lemma 4.2. Thus, $b_{\alpha}$ is a thin Blaschke product.

Remark 4.5. Thin Blaschke products also interpolate on the boundary. In this context, we have a Belna, Cowell, Piranian-like result:

Theorem 4.6 ([12, Theorem 3.3]). Let $\left(\alpha_{j}\right)$ be a sequence of distinct points on $\partial \mathrm{D}$ and let $\left(\beta_{j}\right)$ be a sequence of points on $\partial \mathrm{D}$. Then there exists a thin interpolating Blaschke product $b$ such that $b^{\star}\left(\alpha_{j}\right)=\beta_{j}$ for all $j$.

Thus, given an inner function $I$ and a sequence of points on the unit circle, we may choose a thin Blaschke product $b$ that has radial limit one on the sequence. In this case, our function $I_{\alpha}$ and $I$ will have the same radial limits on this sequence.

One final remark is in order here. Let $M\left(L^{\infty}\right)$ and $M\left(H^{\infty}\right)$ denote the space of nonzero multiplicative linear functionals on $L^{\infty}$ and $H^{\infty}$, respectively, and let $b$ be any Blaschke product. Let $F=\left\{x \in M\left(L^{\infty}\right): x(b)=1\right\}$. Here, we identify functions with their Gelfand transforms. Then $F$ is a peak set for $H^{\infty}$ (with peaking function $(1+b) / 2$ ) and therefore [10, p. 39] $\left.H^{\infty}\right|_{F}=\{f \in$ $\left.L^{\infty}: f\left|F \in H^{\infty}\right| F\right\}$ is a closed algebra with maximal ideal space given by $\left\{x \in M\left(H^{\infty}\right): \operatorname{supp} x \subset F\right\}$, where supp $x$ denotes the support set for the representing measure of $x$. Therefore $x(b)=1$ and so $x\left(I_{\alpha}\right)=x(I)$ for all $x \in M\left(H^{\infty} \mid F\right)$. But $b_{\alpha} g_{\alpha}=b+\alpha(1-b) I$, so $x\left(b_{\alpha} g_{\alpha}\right)=1$ for all such $x$. In particular, $I\left|F=\tilde{b} \overline{b_{\alpha}}\right| F$ and $\overline{b_{\alpha}}\left|F=g_{\alpha}\right| F \in H^{\infty} \mid F$. On every such peak set, then, $I$ is a ratio of such Blaschke products. (This, as well as the Frostman representation, can be compared with the work of D. Suarez [29]).

Axler's observation was the following: choosing $b(z)=z$, in this case, the peak set $F$ above is the fiber $X_{1}=\left\{x \in M\left(L^{\infty}\right): x(z)=1\right\}$. Therefore, the fact that $b_{\alpha}$ has no zero converging to 1 implies that $b_{\alpha} \mid X_{1}$ is a constant of modulus 1 . Thus, $I \mid X_{1}$ equals a Blaschke product on $X_{1}$. Our result can be thought of as the appropriate generalization of Axler's observation.

Acknowledgements. The authors are grateful to the London Mathematical Society and the EPSRC for financial support. We also thank the referee for valuable comments, for improving the proofs, and for providing us with a significant simplification of Theorem 4.4. 


\section{REFERENCES}

1. Axler, S., Factorization of $L^{\infty}$ functions, Ann. of Math. (2) 106 (1977), 567-572.

2. Axler, S., and Gorkin, P., Sequences in the maximal ideal space of $H^{\infty}$, Proc. Amer. Math. Soc. 108 (1990), 731-740.

3. Belna, C. L., Colwell, P., and Piranian, G., The radial behavior of Blaschke products, Proc. Amer. Math. Soc. 93 (1985), 267-271.

4. Berman, R. D., The sets of fixed radial limit value for inner functions, Illinois J. Math. 29 (1985), 191-219.

5. Cantor, D. G., and Phelps, R. R., An elementary interpolation theorem, Proc. Amer. Math. Soc. 16 (1965), 523-525.

6. Cargo, G. T., Blaschke products and singular functions with prescribed boundary values, J. Math. Anal. Appl. 71 (1979), 287-296.

7. Colwell, P., Blaschke products. Bounded analytic functions, Univ. of Michigan Press, Ann Arbor, MI 1985.

8. Daepp, U., Gorkin, P., and Voss, K., Poncelet's theorem, Sendov's conjecture, and Blaschke products, J. Math. Anal. Appl., 365 (2010), 93-102.

9. Fisher, S. D., and Shapiro, J. E., Singular factors are rare, Michigan Math. J. 44 (1997), 231-237.

10. Gamelin, T. W., Uniform Algebras, Prentice-Hall, Englewood Cliffs, N.J. 1969.

11. Garnett, J. B., Bounded Analytic Functions, Pure and Applied Math. 96, Academic Press, New York, 1981.

12. Gorkin, P., and Mortini, R., Radial limits of interpolating Blaschke products, Math. Ann. 331 (2005), 417-444.

13. Gorkin, P., and Mortini, R., Cluster sets of interpolating Blaschke products, J. Anal. Math. 96 (2005), 369-395.

14. Gorkin, P., and Mortini, R., Asymptotic interpolating sequences in uniform algebras, J. London Math. Soc. (2) 67 (2003), 481-498.

15. Gorkin, P., and Mortini, R., Value distribution of interpolating Blaschke products, J. London Math. Soc. (2) 72 (2005), 151-168.

16. Gorkin, P., and Rhoades, R. C., Boundary interpolation by finite Blaschke products, Constr. Approx. 27 (2008), 75-98.

17. Guillory, C., and Sarason, D., Division in $H^{\infty}+C$, Michigan Math. J. 28 (1981), 173-181.

18. Guillory, C., Izuchi, K., and Sarason, D., Interpolating Blaschke products and division in Douglas algebras, Proc. Roy. Irish Acad. (A) 84 (1984), 1-7.

19. Hjelle, G. A., Constructing interpolating Blaschke products with given preimages, Comput. Methods Funct. Theory 7 (2007), 43-54.

20. Hoffman, K., Bounded analytic functions and Gleason parts, Ann. of Math. (2) 86 (1967), 74-111.

21. Hoffman, K., Banach Spaces of Analytic Functions, Dover, New York 1988.

22. Jones, W., and Ruscheweyh, S., Blaschke product interpolation and its application to the design of digital filters, Constr. Approx. 3 (1987), 405-409.

23. Kerr-Lawson, A., Some lemmas on interpolating Blaschke products and a correction, Canad. J. Math. 21 (1969), 531-534.

24. Nicolau, A., Blaschke products with prescribed radial limits, Bull. London Math. Soc. 23 (1991), 249-255.

25. Nikol'skiı̆, N. K., Treatise on the Shift Operator. Spectral Function Theory, Grundlehren der Math. Wissenschaften 273, Springer, Berlin 1986.

26. Royden, H. L., Real Analysis, 2nd edition. Collier-Macmillan, Toronto 1968.

27. Rudin, W., Real and Complex Analysis, 3rd edition. McGraw-Hill, New York 1987. 
28. Stray, A., Minimal interpolation by Blaschke products II, Bull. London Math. Soc. 20 (1988), 329-332.

29. Suárez, D., Approximation by ratios of bounded analytic functions, J. Funct. Anal. 160 (1998), 254-269.

30. Sundberg, C., and Wolff, T. H., Interpolating sequences for $Q A_{B}$, Trans. Amer. Math. Soc. 276 (1983), 551-581.

\author{
UNIVERSITÉ LYON 1 \\ INSA DE LYON \\ ECOLE CENTRALE DE LYON \\ CNRS, UMR 5208 \\ INSTITUT CAMILLE JORDAN \\ 43 BLD. DU 11 NOVEMBRE 1918 \\ F-69622 VILLEURBANNE CEDEX \\ FRANCE \\ E-mail: chalenda@math.univ-lyon1.fr \\ SCHOOL OF MATHEMATICS \\ UNIVERSITY OF LEEDS \\ LEEDS LS2 9JT \\ U.K. \\ E-mail: J.R.Partington@leeds.ac.uk
}

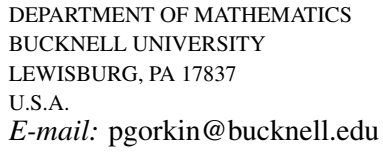

